\title{
Grounded Theory Methods in Child Psychotherapy Research
}

\author{
Michael Rustin
}

\begin{abstract}
This article considers the place of qualitative research in psychoanalysis and child psychotherapy. It discusses why research methodology for many years occupied so small a place in these fields, and examines the cultural and social developments since the 1960s which have changed this situation, giving formal methods of research much greater significance. It reflects on the different pressures to develop formal research methods which arise both from outside the psychoanalytic field, as a condition of its continued professional survival, and from within it, where its main aim is the development of fundamental psychoanalytic knowledge, It suggests that the conduct of mainly quantitative research into treatment outcomes is largely a response to these external pressures, whilst the main benefits to be gained from the development of qualitative research methods, such as Grounded Theory, are in facilitating the knowledge-generating capacities and achievements of child psychotherapists themselves. The paper describes Grounded Theory methods, and explains how they can be valuable in the recognition of hitherto unrecognised meanings and patterns as these are made visible in clinical practice. Finally, it briefly describes five different examples of completed doctoral studies, all of which have added significantly to the knowledge-base of child psychotherapy, and which demonstrate how much can be accomplished using this method of research.
\end{abstract}

\section{Keywords}




\section{Grounded theory, clinical research methods, outcome studies,}

\section{Research Methods in Psychoanalysis: a strange absence}

Psychoanalysis has been unusual in the limited attention it has previously given to what are referred to in other scientific fields as 'research methods'. Whereas most disciplines discuss, evolve and codify their methods of generating and validating knowledge as a substantial part of their activity, psychoanalysis has mostly not done this, or at least, not under the categorisation of research as such. ${ }^{1}$ There is no parallel in the field of psychoanalysis to the large literatures on research methods in sociology, psychology and history, in which alternative approaches, controversies and textbooks abound. Sociology, for example, sought to establish itself as a scientific discipline - seeking to demarcate itself from merely descriptive and anecdotal writings about society, and from rival disciplines such as psychology and economics - by clarifying its own methodological principles, with statistical theory and the techniques of social surveys and structured interviews a central element of this. There is probably no degree course in sociology taught anywhere which does not have a substantial component of research methodology. To an even greater degree, academic psychology has largely defined itself by its claims to scientificity, and by the systematic attention it has given to methods of research.

But despite its apparent indifference to research methodology, the field of psychoanalysis can lay claim to have constituted one of the most productive paradigms of evolving knowledge of the last 100 and more years. Pathways of development of psychoanalytic theories and clinical techniques can be traced back to Freud's initial discoveries, through theoretical lineages such as that of Freud-Klein-Bion and their post-Kleinian successors, and in schools such as the contemporary Freudians, the British Independents, the Relational School in the United States, and equally distinctive developments in France

\footnotetext{
${ }^{1} \mathrm{~A}$ valuable exception was the special issue of the International Journal of Psychoanalysis, edited by David Tuckett, devoted to 'Clinical Facts'. (IJPA 1994).
} 
(influenced by Lacan) and in Latin America. In the literatures which report and codify these developments, the place of what in other fields would be defined as 'research', instead tends to be termed 'technique', reflecting the fact that psychoanalysis is above all a practice-based discipline, in which discoveries are made in the context of clinical work, not in a separate research-dedicated domain of the laboratory or other contexts of investigation. Freud's original discoveries of the unconscious information-bearing significance of dreams and patients' associations to them; of the transference and its later development by analysts such as Strachey (1934); the subsequent recognition of the counter-transference as a source of understanding of patients (Heimann 1950) and Klein's (1926) discovery that children's play could provide a form of access to unconscious mental life in the treatment of children, are all examples of the close links between major new developments in clinical technique and the emergence of new theoretical ideas. Even Lacan's unsettling clinical techniques, refusing the disciplined regularity of other psychoanalytic approaches (and getting Lacan into great trouble with the psychoanalytic authorities) were related to his theory of unconscious mental life and its necessarily disruptive qualities.

Psychoanalysts, including the most original and creative among them, have for the most part believed that knowledge could be advanced through combining deep knowledge and the continuing interrogation of established concepts and theories, with close attention to the phenomena which are revealed to them in their consulting rooms. Belief in this method has been vindicated by its outcomes in the advancement of knowledge. The psychoanalytic paradigm, founded on its core assumptions concerning the significance of unconscious mental processes in psychological development has followed a pattern of 'speciation' of its field of knowledge. This was the term for the process of evolutionary differentiation and 'branching' which Thomas Kuhn (2000) using a Darwinian metaphor, believed characterised the development of most fields of science. Sciences and their methods of investigation differ substantially from one another (Galison and Stump 1996), reflecting the attributes of their different 'objects of study' and it may be useful to consider with which other fields of inquiry psychoanalysis has the most 
affinity. It seems closest to evolutionary biology. Both fields combine subscription to a cluster of fundamental explanatory laws and principles, established in their founding 'scientific revolution (Darwin's in one case, ${ }^{2}$ Freud's in the other), with a 'normal science' (rule-governed practices) which investigate the explanatory powers of these laws for large numbers of instances, always taking account of their particular attributes. ${ }^{3}$ In the Darwinian case, this was, and remains, the study of different species in their interactions with their environments (their ecology). In psychoanalysis, it is the study of individuals, in their specificity, in relation to their human environment, and within the analytic relationship itself. 4

Even psychoanalysts who have been deeply concerned at the refusal of psychoanalysis to engage with the formal protocols of scientific method, and fear its extinction as a result have sometimes admitted that despite its deficiencies, the traditional method of the clinical case-study has been fertile in its capacity to generate theoretical insight and understanding ${ }^{5}$. Thus, against those who have insisted that psychoanalysis adopts methods closer

\footnotetext{
2 The basic principles in the evolutionary case include (a) random genetic variation by mutation (b) competition between the ensuing varieties for reproductive success (c) competitive (and interdependent) forms of adaptations to changing environments. ${ }^{3}$ Scientific ornithology well illustrates this pattern of development. The descriptive observations of bird species with which most people are familiar, if only from natural history programmes on television, are paralleled by meticulously researched quantitative researches, for example studying the effects of crop-changes on the population densities of particular species, or the interactions of predator with predated populations. Bird Studies, the international journal of the British Trust for Ornithology, is notable for its uncompromising commitment to quantitative methods, but its articles remain Darwinian in their theoretical basis.

${ }^{4}$ Here is the memorable conclusion to Darwin's Origin of Species: "Thus, from the war of nature, from famine and death, the most exalted object which we are capable of conceiving, life, with its several powers, having been originally breathed into a few forms or into one; and that, whilst this planet has gone cycling on according to the fixed law of gravity, from so simple a beginning endless forms most beautiful and most wonderful have been, and are being, evolved." The equivalent 'endless forms' in psychoanalysis are the variety and uniqueness of human personalities.

5 "As Peter Fonagy and Mary Target (2003, p. 3-4) have put it "The psychology that Freud discovered and elaborated has enjoyed considerable success as an explanatory framework. This is because its few basic assumptions and propositions are open to endless revision and refinement, and, arguably, because the clinical procedure that provides its evidential base offers a unique perspective on the human mind.' While they urged psychoanalysts and child psychotherapists to adopt research methods compatible with mainstream psychology, they also urged developmental psychologists to learn from the subtlety and complexity of psychoanalytic descriptions and explanations, which their book indeed describes. While there are 'more psychoanalytic theories than we need', there are also 'unique features to each body of theory.' ( $p 2$ 2.)
} 
to the conventional scientific mainstream (in practice this usually means the mainstream of psychology) might one wonder that if the traditional clinical method is not broken, so far as its generative capacity is concerned, how much is it desirable to change it?

Perhaps the 'traditional method' of the clinical case study has been adhered to for so long because it is only by giving attention to the phenomena of the transference and counter- transference that unconscious mental life can be accessed and described. 'Fixes' and improvements to this method may be resisted by psychoanalysts because departure from this investigative method may require compromising their central task, which is to study unconscious mental processes. In other words, methods of understanding, in psychoanalysis as in other fields, are indissolubly linked to their content. The psychoanalyst's concern is that while more conventionally scientific research methods applied to their field may be productive of knowledge of certain kinds, they may lose sight of its psychoanalytic object.

Without prejudging questions of choice between relevant research methods, it is nevertheless clear that it is mainly something extraneous to psychoanalytic understanding itself has been driving the demand for the pursuit of conventionally scientific methods in psychoanalysis. The principal anxiety has been about the absence of evidence for the outcomes of psychoanalytic treatments of mental illness. The question, from health service commissioners, competing mental health professions, and indeed potential clients or patients which psychoanalysts have been under increasing pressure to answer is, 'does it work?' Or indeed how much does it work, and for whom? One can accept the appropriateness of these questions (who would want to practice in ways which have been proved to be ineffectual?) yet note that they are somewhat different from many of those which have routinely preoccupied psychoanalysts and child psychotherapists. These latter are about the nature and functioning of the unconscious mind, the development of personality, failures of psychological development, and the nature of the psychoanalystpatient relationship. These are the fundamental objects of research of psychoanalysis. The clinical case study methods which mainly been used to 
study these matters are sometimes referred to as 'therapy process research' (Midgley 2004 p. 92) although this description risks mistaking a means of investigation for its object. It should however be noted that the investigation of outcomes of a kind has always been central to fundamental psychoanalytic research also, since one sometimes (contentious) measure of whether a true understanding of unconscious states of mind has been achieved is the ways in which patients respond to being understood. ${ }^{6}$

\section{A historical perspective}

The absence of the psychoanalytic field from the field of methodological debate in the human sciences has a cultural and ideological origin. This lies in the attacks made on Freud's own claims for psychoanalysis as a science by philosophers of science such as Karl Popper (1963), and in greater rigour by Adolf Grunbaum (1973), and other fierce critics of the field. (Indeed psychoanalysis is as notable for the hostility of its enemies as for the commitment of its friends.) There appeared to be a discrepancy between Popper's influential model of how sciences advanced, through the 'falsification' and abandonment of hypotheses claiming universal validity, and classic psychoanalytic methods of investigation. In particular, Popper and Grunbaum rejected the idea that patients' response to interpretations could constitute valid evidence for their truth-content. ${ }^{7}$

However it has later become evident that Popper's falsificationist model corresponds to few actual fields of scientific practice, once it became recognised, following the contribution of Thomas Kuhn, a historian of science, that the sciences were more diverse in their attributes than Popper's prescriptive view had asserted them to be. (Puttnam 1975, Stove 1982).

\footnotetext{
${ }^{6}$ One is tempted to suggest that clinical research has been the primary location of psychoanalysis as science, while outcome research is its technology.

${ }^{7}$ Robert Hinshelwood (2015) has attempted to show that it is possible in rigorously conducted clinical research to subject psychoanalytic theories to the Popperian test of falsifiability. For a commentary on his arguments see Rustin (2015).
} 
Within the human sciences, there were different responses to the hegemony of this positivist, or more precisely 'falsificationist', definition of science. Some human science disciplines responded by adopting methods which sought to to comply with the prescriptive philosophers' model of scientific investigation. (Usually this meant privileging experimental and quantitative methods over naturalistic observation and qualitative approaches.) In Britain, the discipline of psychology overwhelmingly chose this route. It thereby won itself a position in the academy as a reputable science, and became widely licensed by the state as an applied 'human technology', to its great professional advantage. Psychoanalysis mostly withdrew itself from such arguments about scientific legitimacy, and chose instead to remain within its enclave of clinical practice, mainly dependent for its economic sustenance on the demand for psychoanalytic treatment by private patients. It functioned as a self-regulated profession rather than as an accredited scientific discipline. As a consequence it had for many years no significant place, in the United Kingdom, in the university systems of undergraduate and postgraduate education and received no governmental support for research. While it retained a significant cultural presence in society, this was more diffused through the humanities - literature, drama, fine art, cinema, popular culture, and philosophy - than the sciences, including the social sciences such as politics, sociology, economics and anthropology. ${ }^{8}$ (The last, the most particularistic and humane of the social sciences, was a partial exception.) Indeed it is often said that psychoanalysis is a hybrid field of knowledge, part science, part humanity, because its concerns are not only with laws of cause and effect, but also with interpretation, with subjective meanings, and with individual differences (Ricoeur 1970) These habits of thought are in contrast to the impersonal and 'objective' standpoint which is deemed to characterise the sciences.

\footnotetext{
8 John Forrester and Laura Cameron's research on the presence of psychoanalysis in Cambridge University in the 1930s has revealing its unexpected influence within scientific circles, but this does not seem to have advanced its scientific status very much. Several of their papers can be accessed at http://www.people.hps.cam.ac.uk/index/teachingofficers/forrester
} 
Different and contradictory pressures came together, from the $1960 \mathrm{~s}$, to change this situation. The expansion of higher education, and the accompanying increase in academic credentialisation gave rise to demands upon many fields of professional education, including the psychotherapies, to obtain academic recognition. The impact of these were even felt in fields like fine art, where traditional practice-based methods of learning were now expected to comply with academic norms in which students' work had to be classified, measured and graded as it was in other disciplines. In the mental health field the Tavistock Clinic was instructed by government in the early 1980 s to obtain academic accreditation for its professional trainings as a condition of their continued central funding. Many psychotherapy programmes were placed under these pressures, sometimes finding that they could make relationships which universities which supported their work, and sometimes that their professional approaches and those of the academy were incompatible. Demands came from students and trainees too. Why, they asked, should years of training in psychotherapy not lead to the same level of academic qualification as a clinical psychology student might obtain? Child psychotherapy and other professions whose development had taken place in institutions like the Tavistock Clinic became victims of their own success. As they came to occupy a larger and better-funded place within mental health services, they were expected to function just like other state-regulated professions.

This was during a period, from the 1980s to the present, when the moral authority and autonomy of many professions had come under political attack. It was argued by their critics that their claims to altruism and concern for the well-being of their clients were merely providing cover for their own occupational self-interest (Le Grand 2006). If formerly passive clients of public services could be transformed into active consumers within competitive markets or quasi-markets, their interests and needs would, it was argued, be better served. Institutions like schools, universities and hospitals became required to compete for their clients with public measures of their relative performance, via 'league tables' and the like, to facilitate 'consumer choice'. But on the other hand, belying the freedom and choice which markets are 
supposed to bring about, much more stringent regulation of provision was imposed, for example through the National Curriculum for Schools, and through batteries of inspections and audits. This system of governance has been described as the new managerialism. (Clarke and Newman (1997) Clarke, J.H., Gewirtz, S, McLaughlin, E. (eds) (2000). The requirement that medical practice should become 'evidence-based', and the role of NICE (National Institute for Clinical Excellence) guidelines in regulating available treatments was the equivalent of this development in the National Health Service. In mental health, this demand began to seriously constrain the autonomy of professionals. It became difficult to obtain recognition of psychoanalytic psychotherapies as fundable forms of mental health treatment, without 'scientific' demonstrations of their effectiveness. These demands, for credentialised postgraduate education, and for demonstrable evidence of treatment outcomes, placed pressure on psychoanalytic psychotherapy. Academically-trained psychologists were at first more adept at meeting these challenges than the psychoanalytically-minded, and the rise of Cognitive Behaviour Therapy reflected this capability, becoming the treatment of choice in the Improved Access to Psychological Therapies expanded programme for mental health which Richard Layard and David Clarke (2015) persuaded governments to adopt. These new norms were especially constraining where, as with child psychotherapy, most treatments were funded by the NHS. Psychoanalysts working mainly in private practice were more insulated from these pressures. This situation, made worse by constraints on NHS funding, is leading to some flight of psychotherapists from the public sector. Progress which had been made in making psychoanalytic psychotherapies available to a broader population with mental held needs is now being reversed.

But while these pressures have sometimes been seen as threatening to psychoanalysis, there have been some creative responses to them. Educational programmes have been developed which remain psychoanalytic in their essence but which have also been able to satisfy academic requirements for rigour and accountability. Similar positive responses have been made to demands to provide evidence of treatment effectiveness. In the 
last few years in Britain, and also, for example in Germany and the USA, several significant outcome research projects have been completed which comply with the requirements of the 'gold standard' of the randomised controlled clinical trial, without compromising an authentically psychoanalytic method of treatment. (Kennedy 2004, Kennedy and Midgley (2007), Midgley and Kennedy (2011).

One should note, however, that the pressures for credentialisation and regulation which have become so prominent since the 1980s were preceded by a more favourable cultural and political climate in the 1960s and 1970s. The field of mental health and its treatment has been influenced both by the radical political culture of the earlier period, and by the conservative reaction to it since the 1970s. Whereas the later 'neoliberal' period has been marked by the imposition of the norms of the market and of restrictive regulation, the earlier period was one of radical cultural challenges to authorities of many kinds. These have been contradictory movements in our culture, ascendant at different times, but each remaining active and in conflict with one another throughout this period.

It was only because there had been radical challenges to the ruling academic orthodoxies in the 1960s and 1970s that creative responses to the later 'credentialising' demands on the mental health professions became possible. Some of the cultural capital which had accumulated in the earlier period, remained available as a resource for resistance when the counter-pressures of a conservative or 'neoliberal' kind made themselves felt from the 1980 s. $^{9}$

Challenges to the dominant stratified and authoritarian culture were in part a consequence of the huge educational expansion which had taken place since the war. This allowed hitherto subordinated and unheard fractions of the population to find a visible cultural presence and public voice. Those who originally authorised the mass expansion of the educational system, including

\footnotetext{
${ }^{9}$ Nick Midgley (2006) has noted that at the beginning of his research career, qualitative methods were excluded from mental health research, but that in later years their relevance became accepted.
} 
university education, may have anticipated that the existing cultural hierarchies would merely be reproduced, but on an extended scale. But this is not entirely what happened. New unruly voices began to be heard, and dissensions emerged around the axes, for example, of class, gender, generation, ethnicity and sexuality. ${ }^{10}$

The sciences themselves were affected by conflicts over authority and legitimacy. Thomas Kuhn's 'Structure of Scientific Revolutions' (1962) published at the beginning of the 1960s, was pathbreaking, since it recognised that scientific investigation itself had historically been subject to contestation and change. The sciences were shown to be social constructs, like other forms of symbolic representation. Kuhn's concept of changing 'paradigms' of knowledge was immensely liberating to researchers in many fields where restrictive orthodoxies had held sway, and in which theoretical and methodological innovation had been forcefully discouraged.

Psychoanalysts were among those who saw the relevance of Kuhn's ideas for their work - a search for 'psychoanalysis' and 'paradigm' on the internet generates 50,000 references.

The field of sociology well illustrates this pattern of liberated development. In the 1960s and 1970s the subject expanded rapidly. Increased interest in it expressed the desires of students, many of them the first in their families to go to university, to understand their rapidly-changing and contested society, and their own position as mobile individuals within it. (The experience of the working class 'scholarship boy' described by Richard Hoggart (1967), was emblematic.) As the discipline grew, especially within new universities and polytechnics, challenges to its previous 'positivistic' orthodoxies emerged, leading to a sometimes bewildering proliferation of theories and methods. One such perspective was that sociologists should investigate and report the experiences of social actors from their own subjective points of view, and not merely 'from above', from the perspective of society's government and policy-

\footnotetext{
10 Demands to return to educational 'basics' and to return to a more traditional curriculum have been made by conservative educationalists from the 1970s onwards, in the first instance by the authors of the Black Papers. These arguments have had a considerable influence on the educational policy of successive governments. On this history see Ball (2013).
} 
makers. Just as American popular culture had challenged the norms of British 'high culture', and created assertive new forms of expression especially attractive to the young, so the sociological perspective of 'symbolic interactionism' in the United States gave social scientists in Britain interpretative research methods by which they could study education from the point of view of low-stream children, or delinquency and crime which took account of the experiences of delinquents. Many disciplines were influenced by this more democratic climate. 'Cultural studies' emerged, in competition with traditional humanities disciplines, and gave legitimacy to the study of popular culture. A radical genre of 'history from below' developed, which studied the experiences of subordinated, often working class, people. Even in a field as conservative as military history, a leading historian such as John Keegan (1976) wrote about war not only from the usual point of view of generals and statesmen, but describing the experience of the ordinary soldier. Clifford Geertz's (1973) cultural anthropology, with its emphasis on the interpretation of cultures - the meanings created by social actors themselves - and even the later Wittgenstein's refocusing of philosophy on different 'forms of life' - can also be seen as aspects of a democratisation of intellectual inquiry.

In an unexpected way, this new climate was congruent with a fundamental principle of psychoanalysis. This is that the starting point for all psychoanalytic understanding is the experience of the patient, as this is communicated to the analyst or psychotherapist in the transference situation. While psychoanalysis has from the beginning evolved a technical language for use within its scientific community, it has always been essential to its practice that its insights be communicable to patients in the ordinary language of human emotions. (This is particularly necessary in the psychoanalysis of children.) A precondition of any psychoanalytic research method is that it must take account of the 'subjective' dimensions of experience, not only of the patient but of the analyst too. What differentiates this from a parallel insistence on the dimensions of meaning and subjectivity in other social sciences, and continues to cause friction with even with their more interpretative schools, is its belief that these have an unconscious dimension which is by definition not 
readily transparent to investigation. Although psychoanalysis in many respects is not at all populist or egalitarian in its assumptions (it often endorses certain kinds of innate authority and difference, for example those related to the 'facts of life' of generation and gender ${ }^{11}$ ) it is nevertheless (by intention at least) respectful of every individual's mental life, in a way which differentiates it from more 'objectifying' kinds of psychology and psychiatry.

\section{The generation of knowledge in child psychotherapy}

This heuristic starting point in the experience of the person, as this is revealed within the therapeutic relationship, is what has given rise to psychoanalysis's reliance on the clinical case study as its primary source of empirical data and understanding. There are few theoretical developments in psychoanalysis, at least in Britain, which do not make reference to clinical instances or case narratives in their justification.

British psychoanalytic writing, and writing in child psychotherapy, appears to possess a 'deep grammar', whose recurrent features are:

(a) the setting-out of a relevant field of concepts and theories, most often sourced to specific canonical, or exemplary texts

(b) a description - often very brief - of the background of a clinical case

(c) an exposition of some segment of clinical material from a case or cases, from which is inferred the presence of an explanatory structure or process set out in the terms of psychoanalytical theory

(d) a conclusion explaining how the instance(s) give evidence for and exemplify addition to knowledge which the particular paper is purporting to provide.

\footnotetext{
${ }^{11}$ Money Kyrle's are the best formulations of this principle. $(1968,1971)$.
} 
However, the relationships which exist between source concepts and theories, clinical material, and the conceptualisations which emerges from them, are varied and complicated. There is a study to be made of such variations of form. In some key papers, clinical material has been set out which an analyst demonstrates is inconsistent with the received theoretical assumptions which she had taken as her initial frame of reference. The material, or 'clinical facts' identified within it, are then cited as grounds for a departure from or extension to the established theory.

Here are three examples of this way of announcing a new psychoanalytical discovery. One is Klein's (1926) demonstration of the significance of unconscious phantasy in the early life of the child, through the use of the technique of 'play therapy'. A second is Herbert Rosenfeld's (1971) distinction between 'libidinal' and 'destructive narcissism', thus bifurcating the pre-existing concept of narcissism, and mapping the two parts on to Freud's dual concepts of the life and death instincts. Rosenfeld grounded this in his description of a patient whose analysis only made progress (that is, gave rise to a new flow of thoughts and feelings) once he had recognised that this new category, of 'destructive narcissism', corresponded to what he was now observing and experiencing. A third example is Paula Heimann's (1950) description of her first recognition of the knowledge-bearing significance of the counter-transference, as she explored with a patient the hypothesis that the persisting disturbance which she was experiencing in her work with him reflected her patient's mental disturbance more than her own, as she had at first feared was the case.

This exemplary genre of theoretical advance is paradoxically quite close to the 'falsificationist' model according to which psychoanalysis, according to Popper, had failed to meet the criteria of an authentic science. In these instances it does seem that hypotheses derived from existing theories were tested and abandoned specifically because they were disconfirmed by clinical evidence. Alternative hypotheses were then adopted which better corresponded to the clinical facts. 
In most psychoanalytic publications which have advanced knowledge, however, the relation between clinical evidence and theoretical development is by no means as clear-cut as in these instances. A great deal of clinicallybased writing aims to demonstrate, how the development of a personality formation, or a specific therapeutic difficulty, can be explained by reference to concepts and theories which are already known and accepted. Often it is the description and interpretation of a clinical instance which is given priority, not the questioning or modification of an existing theory. Its relevance is in providing additional empirical evidence of validity. Because psychoanalytic investigations are usually made in clinical contexts on a 'case-by-case' basis, theories are mainly tested through the sequential accumulation and comparison of such cases. ${ }^{12}$ These usually take place over a period of time, and through the work of different practitioners. This is how promising new ideas, first often put forward by leading figures in the field, have been exposed to extensive clinical tests. These 'tests', informal as they usually are, may lead to the discovery of new ideas or theories, and/or their elaboration to take account of new phenomena.

An example of this second pattern of discovery is the understanding of the psychopathology and therapeutic treatment of severely deprived children, which emerged as an area of practice central to child psychotherapy in the 1970s, and has developed since then. ${ }^{13}$ Certain key theoretical ideas, drawing in particular on Klein's theory of the paranoid-schizoid and depressive positions, and on Bion's theory of projective identification and of the container-contained relation, were brought together by child psychotherapists to make sense of the troubled and often violent internal worlds of children whose early caring relationships had been grossly deficient or abusive. Child psychotherapists sought to understand the consequences of these internal states of mind for their patients' relationship with their carers, often by inference from their experience of the children in their own

\footnotetext{
${ }^{12}$ Nick Midgley (2004,p. 92) has usefully described this process of inference as 'generalising to a theory'.

13 The starting point of this development was Gianna Henry (later Williams)' paper 'Doubly Deprived' (1974), then followed by the collection of papers edited by Boston and Szur (1983). Many significant papers have followed, mainly published in this journal. A recent collection is Andrew Briggs' (ed) Waiting to be Found (2012).
} 
transference relationship with them. The prominence of such children in the case-loads of child psychotherapists in the NHS arises from the fact that these emerging understandings and techniques gave rise to a capacity to work therapeutically with them more effectively than other mental health professionals.

This sub-field is an example of the way in which core psychoanalytic ideas have been both tested out, case by case, and elaborated to take account of variations in patterns of psychological development. It is an example of 'speciation' in which a sub-field of psychoanalytical theory has advanced in a branching pattern, each sub-branch describing a specific variation. Examples are developments which arise from the absence of fathers, parental addiction to drugs, domestic violence, sexual abuse, or circumstances where a lookedafter child has been subject to many changes of placement, or adoption. One can trace similar evolutions from a 'core theory' in other areas of clinical specialism, such as autistic or Aspergers personality formation, or concerning the unconscious concomitants of physical or mental disability. ${ }^{14}$

The fact that knowledge is advanced in psychoanalysis through the understanding of variations and differences -for example in patterns of development, personality formations, mental disorders, and therapeutic techniques - works against its theoretical unification. The need for psychoanalysis (and child psychotherapy) to have fewer theories, but more powerful ones better supported by empirical evidence, has been forcefully advanced by critics of the traditional case study method by influential psychoanalytic researchers such as Peter Fonagy (2009), Robert Wallerstein (2005) and David Tuckett $(2000,2005)$. The idea is that such unification

\footnotetext{
14 The Tavistock Clinic Karnac book series includes many examples of edited collections of papers which report developments in these various sub-paradigms of child psychotherapy theory and practice. Even though most of these make little reference to formalised methods of research, one should surely these collections as the reports of a successful ongoing child psychotherapy research programme. Indeed given its evidential basis in clinical practice, one can equally view the development of the whole corpus of psychoanalytic theory as the outcome of a multi-faceted research programme. It is important than the designation of 'research' should not be confined to those practices which conform to the norms of clinical psychology, which often provides the implicit model of scientific legitimation in this field, but should respect the outcomes of the 'normal science' of psychoanalytic clinical practice.
} 
would bring psychoanalysis closer in its theories and methods to other scientific fields, some of which are able to work at much greater levels of generality. However it seems that one could pay a high price for theoretical unity, in the disappearance of the variety and difference which make some fields interesting and theoretically productive in the first place. Theoretical developments in psychoanalysis often do not conform either to purely 'deductive' (i.e. logical) models of explanation, or 'inductive' ones (inferences from experience). They may combine both dimensions, or arise unexpectedly as a consequence of an intuitive insight by a psychoanalyst. Such intuitions are commonly part of scientific investigations.

Metaphors, as Ronald Britton (2015) has pointed out, may have a vital role in creating new understandings in psychoanalysis A metaphor, according to the classical formulation by Max Black (1962) is a formulation which asserts an equivalence between the properties of a field whose attributes are already understood, to one where this is not yet the case. ${ }^{15}$ (One can think of this relation as between target and source). An example of the power of metaphor can be seen in the theory of the container-contained relation first put forward by Bion. The crucial equivalence (metaphors make a more immediate conceptual leap than assertions of perceived similarity) was between the projective processes observed by Bion in his work with psychotic patients, and the projective unconscious exchanges which take place between mothers and their babies. This may have been essentially a leap of the imagination by Bion, since he is not known to have worked either clinically or as an observer with mothers and infants. His insight developed from Klein's earlier discoveries about early mother-infant relations, and perhaps also from what he may have learned from Esther Bick with whom he was in close touch about the infant observation method which she was pioneering. Bion's recognition of the significance of the container-contained relation, and of the projective and introjective processes which it framed, proved to be highly

\footnotetext{
15 Thus the concept of a computer virus makes use of our previous understanding of biological viruses to understand many attributes of these 'infections' of computer software. The reason why this metaphorical equivalence works so well is because biological viruses are themselves essentially bearers of information, liable to reproduce themselves within an appropriate host.
} 
significant for the understanding of unconscious mental life in many different contexts, for example those of infancy, the consulting room, child development, and indeed of institutions.

\section{Grounded theory in child psychotherapy}

The pattern of knowledge-generation which I have described above preceded the development of formalised methods of data analysis, such as Grounded Theory or Interpretative Phenomenological Analysis (IPA), which over the last twenty or so years have become widely used, in part as a consequence of the development of doctoral studies in psychoanalysis. ${ }^{16}$ It is a requirement of study ${ }^{17}$ for doctoral degrees, whether PhD or Professional Doctorate, that an explicit research method is set out, and that research findings can be shown to have been generated or validated through the application of such a method.

The affinity between Grounded Theory methods and qualitative research in psychoanalysis lies in approaches both to the collection and the analysis of data. Grounded Theory methods were originally developed by sociologists to understand data gathered in the form of open-ended observations, and relatively unstructured interviews. It was essentially a naturalistic method, aiming to capture the complexity of social life as it takes place. In this respect it was distinct from the methodologies of contrived experiments, or structured social surveys whose design incorporates prior theorisations and hypotheses about a chosen object of research.

Psychoanalysis, and its associated observational methods of study, is also in its own way 'naturalistic', in that its primary materials for study are the

\footnotetext{
16 David Tuckett (1994) was an early advocate of the use of Grounded Theory methods in psychoanalysis.

17 Grounded Theory and IPA are comparable in their prescription of methods of line-by-line or segment-by-segment coding, and thus to a degree may be interchangeable methods for analysing data. However they differ in so far as the objective of Grounded Theory is the development of theory, while that of IPA is more the elucidation of actors' own understanding of a situation. Grounded Theory emerged from sociology, a strongly theoretical discipline, IPA from psychology, which has usually adopted a more minimaliist theoretical approach to its objects of study.
} 
communications which are made to analysts in settings which have definite boundaries, but which otherwise leave a great deal of space for patients to set agendas and provide communications (of different kinds) which it is the work of psychoanalytic therapy to understand. Thus a research method which expects that meaning will emerge from data which has little predetermined content or shape is appropriate for psychoanalysis as well as for naturalistic kinds of sociological or anthropological study. In this respect it can be contrasted with the method of an experimental research design such as the attachment theorists' 'Strange Situation Test.'

In regard to the analysis of data, Janet Anderson (2006) has pointed out that Grounded Theory has been adopted as a research method by many child psychotherapist researchers because its analytic procedures are so close to the 'line by line' practice of supervision through which they have previously learned to reflect on the meanings of clinical material. The ideas of line-by-line analysis, and of conceptual and theoretical coding set out by the methodologists of Grounded Theory, thus turned out to closely mirror the practice which experienced psychoanalytic supervisors carried out orally in dialogue with their trainees. They are indeed, as Anderson pointed out, "wellsuited partners."

The advantage of the Grounded Theory protocol over oral supervision is that its inferential steps, from transcripts of session records, to summative theoretical concepts and categories, are required to be set out in both narrative and tabular form. They are in this way made transparent and 'accountable' to readers, line by line, segment-by-segment or session by session. Presentations of this method also require that researchers justify their selection of clinical material for analysis, by explicit criteria. A radical form of selection is usually necessary since the volume of clinical material generated by intensive clinical cases is far larger than can be presented in its totality. Criteria of selection may differ - examples are sampling of different periods in a therapy, focus on moments of especial clinical intensity, and 'turning points', - but the central requirement is that the criteria must be explicitly justified. This presentation of clinical material and the theoretical 
inferences drawn from it makes it possible to improve on the much-criticised practice of illustrating arguments with 'clinical vignettes', whose relation to a larger clinical record might be poorly explained. ${ }^{18}$ Analysts have always used their clinical judgement to decide whether claims made for the significance of case material are justified or not, and their judgements were often sound. But since in more academic research contexts such 'subjective' judgements are deemed to be insufficient or unreliable, the greater transparency of expositions based on Grounded Theory offer a signficant methodological gain.

One early difficulty in the adaptation of Grounded Theory methods to psychoanalysis has been overcome through the softening of an early 'antitheoretical' demand by Grounded Theory's pioneers that empirical data should be collected and analysed witout theoretical preconceptions about their possible meaning. As the Grounded Theory method was adopted by disciplines beyond its original field of sociology, it became recognised that some theoretical assumptions, however implicit, are likely to define and influence every investigation (Charmaz 2006). Thus there is no such thing in research as a tabula rasa, since data cannot be identified and specified without some criteria of selection. There is no reason for psychoanalysis to be an exception to this principle. The requirement of Grounded Theory is not to have a blank mind in the face of data, but rather that a researcher should hold in abeyance her expectations about possible meanings, and to allow inferences to emerge from the encounter with the material itself. Similarly, when Bion asked psychoanalysts to 'eschew memory and desire' on entering a clinical setting, he surely did not mean that they should forget everything they had previously learned about psychoanalysis. It is clear that Bion's own clinical understandings were informed by his own deep theoretical knowledge.

\section{Outward-facing and inward-facing research in child psychotherapy}

A useful distinction can be made between the demands for scientific credibility which are made upon psychoanalysis from outside its field of practice, and

\footnotetext{
18 The use of clinical vignettes as validation is one of the most strongly criticised aspects of psychoanalytic case presentations. See for example Spence (1994).
} 
those which arise from within it. 'Outcome research' is for the most part a response by the psychoanalytic profession to demonstrate that it can meet the requirements of 'evidence-based medicine' which are now being imposed on practitioners. (The redistribution of authority is from semi-autonomous professions to the regulatoary state.) From this perspective the specific psychoanalytic processes through which outcomes are achieved are of little interest. In effect, the psychoanalytic interventions themselves are 'black boxed' as scientists put it. (Latour 1983). What is being measured are the effects of uniform and replicable treatments, while little significance is attached to the theories which may underpin them. Comparability (if not uniformity) of inputs is essential, and it is this that treatment manuals aim to make possible. ${ }^{19}$ The requirement for rigorous methods in this form of research is principally to give credibility to the outcomes which can be claimed to have occurred. ${ }^{20}$

The value of more rigorous qualitative research methods, such as the use of Grounded Theory, is of a different kind. Here the purpose is to advance the production of 'fundamental research' in psychoanalysis, to make new discoveries which are primarily of interest to the scientific community of psychoanalysts and psychotherapists, and to other practitioners (such as social workers) who may share their understandings. The benefits to be derived from the improvement of qualitative research methodologies of this kind are largely endogenous to the field, contributing to its own development of theory and technique.

\footnotetext{
${ }^{19}$ A manual setting out guidelines for the Short Term Psychoanalytic Psychotherapy intervention developed in the IMPACT study is currently in preparation.

20 However, the existence of data-sets reporting treatments of significant numbers of cases, where both the sample of cases and treatment methods have been standardised to provide a degree of uniformity and comparability, is a valuable resource for fundamental psychoanalytic research. It is noteworthy that the IMPACT study has already given rise to the adoption of its Short Term Psychoanalytic Psychotherapy model of treatment within child psychotherapy training, since it was found (even in advance of the outcome data from IMPACT being known) that this treatment protocol was especially appropriate for working with adolescent patients. Another researcher (Miriam Creaser) using this data has been comparing audio transcripts of clinical sessions with the post-facto written notes made by the therapists, on which clinical researchers have hitherto mainly relied.
} 
In the short term at least, it seems unlikely that such developments will have much direct bearing on the 'external' scientific reputation of psychoanalysis. The widespread intellectual and dispositional resistances to the recognition of unconscious mental phenomena seem unlikely to be dispelled merely by the use of rigorous qualitative methods (such as those of Grounded Theory) to analyse clinical records. The heuristic value of Grounded Theory (and of similar methodologies) to psychoanalysis is likely to be felt more within the field, than in its relations with those outside it. It can enhance the quality of fundamental psychoanalytic research and enlarge the community of psychoanalytic researchers.

However one benefit that the use of such qualitative methods has conferred on the psychoanalytic field is the recognition of the legitimacy of psychoanalytic research, for example in making possible the undertaking and assessment of doctoral studies. The adoption of the standard protocols required for the validation of theses - systematic reviews of research literatures, the explicit formulation of research questions, and the adoption of recognised method of research - has made psychoanalytically-informed research methodologically comparable to research within different theoretical paradigms. This has been a significant advance for the field, and may in due course lead to a wider interest in its substantive findings and discoveries.

The 'theory neutrality' of the Grounded Theory methodology has from this perspective been valuable. Its founders (Glaser and Strauss 1967) prescribed that theory should emerge from data by a process of induction, contesting the doctrine that scientific research practice should consist of the empirical testing of theoretically-derived hypotheses. ${ }^{21}$ The inductive approach of Grounded Theory made less likely the disqualification of research for reasons of theoretical antipathy, since according to this model, theories are only useful in so far as they emerge from and give meaning to actual data.

\footnotetext{
21 The Grounded Theorists in the 1960s were challenging the theoretical hegemony of structural functionalism in American sociology at the time, but also seeking to develop a method that was as empirically rigorous that of the influential quantitative methodologists of the time.
} 
As the use of Grounded Theory has developed, especially in the social sciences, the anti-theoretical bias which might have imposed a constraint on psychoanalytically-oriented research has been modified by its leading practitioners. Later advocates of the method, such as Charmaz (2006) have acknowledged that in practice all researchers bring some theoretical preconceptions to their field. Psychoanalytic researchers thus need be no different from those working in other paradigms in the way in their relation to their object of study. It has thus proved feasible to explore questions (or 'solve puzzles') which can be characterised as the 'normal science' of the psychoanalytic paradigm (or that part of it occupied today by child psychotherapy), while at the same time making use of the primarily inductive, or more accurately abductive, ${ }^{22}$ research method of Grounded Theory. This is a rather unusual conjunction.

The evidential problem which psychoanalytic researchers have had to confront arises from the central presupposition of their field, namely that unconscious mental states exist and exercise substantial causal powers over human thoughts, feeling and actions. Since almost by definition, unconscious states of mind are not transparent to observers, or indeed to the self, and are known only by inferences from their effects, this has always constituted a methodological challenge for researchers.

A common starting-point for such investigations is where unexpected conjunctions of behaviour have occurred, but where explanations fail or are insufficient unless they are able to take account of unconscious states of mind. Some of the most significant discoveries of clinical research in child psychotherapy have been of this kind. Why, for example, do adoptive placements with apparently loving and well-balanced families sometimes encounter severe difficulties? It has been found that explanations in terms of the 'internalised beliefs' of adopted children, regarding the expected behaviour of parental figures, are the most adequate to this situation. This is especially the case where it is found that such beliefs can be modified through 
being brought to conscious recognition in psychoanalytic therapy, and where as a result improvements in actual relationships ensue. One way of putting this, adapting the sociological formulation of Max Weber (1978), is to say that investigation of the unconscious provides 'explanation at the level of meaning', adding to the 'explanation at the level of cause' which is achieved through the evidence of the constant conjunction of facts.

It is usually a situation of 'excess' (Zizek 1990) which creates the space in which psychoanalytic explanation is found necessary. That is say, it is called for when what is happening seems to be beyond the limits of commonsense understandings. After all, the main subject of psychoanalytic attention has from the start been states of mind and behaviour which appear to lie beyond reason. It in this interesting space that psychoanalysis makes its entry, whether in the case of individual subjects or social states of mind.

It is because literature and the arts have been more willing to explore particularities and extremities of mind, feeling and personality, that they have an affinity with psychoanalysis which most of the sciences have lacked. The idea that the complexity of the mind is best traced through symbolic connections and equivalences has always been well understood by the writers of poetry, drama and fiction. Imaginative literature and psychoanalysis both recognise that the understanding of minds depends on the interpretation of meanings, and not merely on the correlation of observed facts. Many of the connections explored in the 'grounded theory' analysis of clinical texts are those involving symbolic and metaphoric meanings, with patients' actions in the clinical setting understood in terms of what they signify and intend, often unconsciously. The causal efficacy of a mental act (for example in making someone feel or behave in certain ways) can only be understood if attention is given to its 'subjective meanings' to those concerned.

There is now a significant amount of completed psychoanalytical research which has made use of grounded theory methods (or of Interpretative Phenomenological Analysis). Some of this has taken place in the context of doctoral programmes in child psychotherapy (and other disciplines such as 
social work) at the Tavistock and Portman NHS Trust. The opportunity for clinicians to re-examine, as doctoral researchers, the records of their own sessional material (and other information surrounding a case) subsequent to the completion of their clinical work, has brought about new understandings and discoveries. The systematic 'grounded theory' analysis of clinical data has made visible latent patterns and meanings which had often not been recognised while the clinical work was proceeding. In supervision the priority will often have been to understand the immediate interactions and needs of a patient and the therapist - with difficult patients these can be intense - and not with wider questions of theory or technique.

The line-by-line supervision of a clinical case usually involves the detailed consideration of particulars - for example specific patient-therapist interactions. It slows down the action, so to speak, so that it can be examined more closely. Subsequent grounded theory analysis of the material creates an additional space for reflection. It allows - indeed requires - the systematic study of an entire clinical record, session by session. Its 'constant comparative method' prescribes the generation of codes, categories and ultimately concepts and theories (in ascending order of generality and transferability) from the clinical material itself. The requirement that there should be explicit criteria for the selection of clinical episodes for detailed study makes the research process accountable and transparent, lessening the risk that findings may merely reflect a clinician's desires or prejudices. ${ }^{23}$ The starting-point for such a selection may be a therapist's memory of particularly intense, painful, or even epiphanic episodes, or of the impact on her of a particular case. However the requirement to describe and locate these in a specified conceptual field adds to them a dimension of objectivity, and enhances their value as evidence.

The tabular notation often sometimes favoured in Grounded Theory analysis, is often an aid to inference, connection, and discovery. Usually, the first

23 Britton (1998) describes this risk as it arises in analytic work itself. 
column is the transcribed or literally-recorded clinical material itself, its segments following downwards in temporal sequence. Subsequent columns, which may be labelled codes, categories and finally concepts, are set out in rows from left to right. This tabular format enables concepts to be related to the material from which they emerge, and sequences of clinical descriptions to be interrogated for previously unrecognised meanings. It may be illuminating to use separate columns for the description and words of patients and therapists, to map their interactions. Patterns, for example of regression, repetition or therapeutic progress, can be identified, and the heuristic value or 'groundedness' of theoretical conjectures tested against the evidence for them, there on the page. Of course, distinctions of these kinds are commonly made in the narrative analysis of clinical sessions, with segments of clinical material followed by analytic commentary on their meaning. However, some have found that tabular representations of the analytic process can aid understanding, just as Bion's 'grid' was found useful by him and others in defining different levels of reflective capacity in the mind. It may happen that in setting out a notation to represent a clinical process, a researcher may lose contact with its inner 'music', as perhaps can be the case in the study of a musical score. Grounded theory analysis at its best is not a mechanistic procedure, but enables its practitioners to recover and re-imagine their original clinical experience.

\section{Research using grounded theory: some examples}

There are a number of examples in the published child psychotherapy literature of clinical studies which have advanced knowledge with the aid of the practice of grounded theory analysis. Nearly all of these had their origin in doctoral research, subsequently written up for publication. There are now also many completed doctoral theses, accessible through libraries, which one hopes will soon become available for wider readerships..

I will briefly summarise a few of these examples. Marguerite Reid (2003) analysed the clinical record of her work with vulnerable mothers who had earlier lost a baby, and whose states of mind during a new pregnancy were a 
source of concern. Her grounded theory analysis of her clinical record revealed that the unconscious configuration which was giving rise to anxiety was connected to unresolved mourning for a lost infant. The therapeutic intervention which enabled this to be worked through made it possible to accept the potentiality of a new alive baby, taking the place of the dead baby of phantasy. It was notable that the researcher found herself drawing on the core ideas of Freud's Mourning and Melancholia as she sought to give theoretical meaning to her clinical material. Subsequently Reid has found that the ideas evolved in her clinical study have had a fruitful application in reflections on certain works of literature, where the consequences for individuals of early exposure to the states of mind of mothers who had been bereaved through loss of an earlier child, have been explored. Reid also found that her research had a significant clinical impact, enabling her to gain support for the establishing of a perinatal psychotherapy service for mothers found to be at risk for this reason.

Janet Anderson's (2003) study of children who engage in extreme risk-taking behaviour, was also based on a Grounded Theory analysis of her clinical case records, as she herself has described. This was an instance where the emergence of the ideas which gave meaning to cases which had at first sight seemed more different than similar came from intensive analysis of the material. More mechanistic kinds of factorial analysis had failed to establish a coherent explanation, when she recalled the more holistic pattern which Freud had recognised in Sophocles tragedy of King Oedipus, and perceived, in a remarkable serendipitous intuition, its relevance to her sequence of clinical cases. The central issue was the troubled relationship of a child to a parental couple, and the several different unconscious constellations of mind which could influence this. Oedipus has been put out to die, as a baby, because of a prophecy (unconscious phantasy) that he would grow up to kill his father. Anderson's creative conjecture was that a child engaged in risktaking behaviour might feel himself to have been denied his parents' love, and metaphorically left out to die, the terror of which was being perhaps enacted in quasi-suicidal risk-taking. Anderson developed a concept of 'havens' to characterise these relationships, One of these was the phantasy of expulsion 
and exposure, which she termed 'no haven'. Another configuration was where anxiety concerning unconscious feelings of hatred led to a mother's total possessiveness and reluctance to separate from a son (all of these risktaking patients were male, in this particular sequence of work.) This led certain boys to be possessed by an illusion of invulnerability, leading them to take risks while in a state of denial, but at the same time enacting the disavowed destructive anxiety of the parents (mother in particular) which had been projected into them. (Anderson termed this state of mind, 'illusory haven'.) It is striking that, as in Reid's work, illumination came, in this case in an unforeseen way, from Anderson's recalling the theory of Oedipal desires and anxieties which has always been fundamental to the psychoanalytic paradigm. Anderson's work has a significant implication for clinical assessment and practice, in that it proposes that attention be given to the unconscious phantasies active within those families where children are found to be enacting behaviours dangerous to themselves.

A third example is Jenifer Wakelyn's study of the experiences of an infant who was placed in foster-care from birth, with a view to an eventual adoption which did indeed take place at the end of a year. Her role was as a 'therapeutic observer', that is to say as an observer who both made use of the methods of psychoanalytic infant observation long established at the Tavistock, and now widely followed elsewhere, and who took a more active role in facilitating the family's experience of fostering this baby. Wakelyn became particularly interested in the relationship between the baby, the family and the system of local authority care which held the formal responsibility for the baby's wellbeing. From her grounded theory analysis of the data, concepts emerged which gave meaning to the emotional experiences of all those involved in the situation. Her key concepts - they are metaphors - were Tornado, Machine, and Limbo, to characterise states of disorganisation and confusion surrounding surrounding the infant, and Matrix to describe a state of containment and connectedness. These ideas convey the intensity of the emotional force-field to which the infant and his temporary family were exposed. This is highly relevant to the understanding of the situation of looked-after children, but is often overlooked as individuals and social 
systems defend themselves against the anxieties, conscious and unconscious, which might otherwise be aroused by emotional contact with the traumatic situation of such children and their families. The value of this work is on the one hand to show how insensitive professional/ bureaucratic networks can be in the performance of their tasks. The proposed hand-over of the baby to its prospective adoptive parents, hundreds of miles away, was for all the world as if it was merely a parcel, until an intervention was made to conduct this in a more sensitive way. On the other hand, its value is to show that the reliable presence of a psychoanalytic informed observer can itself provide emotional containment to protect a family from the turbulence around it, even more where an observer role has been extended in a therapeutic direction.

A fourth example is of the use of the grounded theory method to reflect on a psychoanalytic infant observation. Wendy Shallcross's doctoral thesis was about the first year of life of a child whom she originally observed as a student many years before she decided to make this the subject of her research. The observation had several distinctive features, including a serious accident befalling the infant's mother, her consequent separation from her baby for a period, until she returned home from hospital, while the observation continued on its weekly basis. Among the issues to which Shallcross (2012) chose to give attention was the development of the infant in its first four weeks of life. She formulated the view, through close attention to the detail of what she had seen, and had described each week in her exceptionally finegrained notes, that the infant had developed a far more active and curious relationship with his environment than conventional accounts of infant development specify. She also came to believe that a disposition of the infant to defend himself against the overwhelming intensity of feelings aroused in him by his experience of mother and her breast, visible in the earliest movements of his hands, could be seen to be a precursor to the later defences he adopted when exposed to the trauma of his mother's absence, and of her subsequent return in what seemed to him (at first) to be a quite damaged condition. She saw this response as an instance of the 'aesthetic conflict' which Donald Meltzer (1988) has argued is a recurring aspect of an infant's relationship to mother's breast. One pleasing aspect of this study is 
that although it describes a nearly-catastrophic event in the life of the family, whose distressing circumstances are described by the researcher, the family was able to resume a benign course of development, which the observer was able to record during the later part of her two year observation.

The final example of research I will give is so far available only in its doctoral thesis form. Marie Bradley undertook an intervention in the child care system, whose purpose was to explore whether a child psychotherapy procedure of assessment, undertaken over just four sessions, could provide an understanding of the state of mind of children awaiting placement which would be deeper and more informative than that usually available to the professional networks charged with making such decisions. This was in response to recognition of the fact that decisions about the placement of children were often being made with insufficient knowledge of their states of mind, or even history, and that in general children themselves seem to have little presence or voice in decisions which are fundamental to their well-being.

Bradley discovered that a great deal could be learned from these four-session assessments, whose essential procedure was to allow each child a space to express his thoughts and feelings not through being interviewed or asked questions, but through being given the kinds of simple materials for play and drawing of which child psychotherapists make use in the clinical setting. The hope was that in this setting the children might able to communicate their states of mind, hopes and expectations, in their own way. Bradley found that this was indeed what took place, and that she learned a great deal about the children she assessed, including aspects of them which were unexpected. Children who had appeared quite defended and resistant to human contact, in a more formal situation, were able to express themselves in this context, and to intuitively recognise that the therapist they were meeting was interested in and capable of understanding them. She also found that the network responsible for decision-making were responsive to the assessments she provided, in particular because of the detailed descriptions she could give of what the children had actually said and done in their four meetings with her. 
Specifically relevant from the research being considered in this article was Bradley's experience that it was the analysis of her own assessment record using a Grounded Theory method which enhanced her understandings of the children. She had written a record of each assessment session in as much detail as she could remember, immediately after it had taken place. Child psychotherapists are trained over many years to write such reports from memory. It was the line-by-line analysis of the material which had revealed the depth of meaning it contained. For example, it was the appearance, in an apparently tough and defended boy, of quite tender feelings and identifications, in his play with the toy animals and people she had provided, which made her recognise this child's capacity and need for affection. This made it possible to consider different placement and therapeutic options for this child than might have been found appropriate if more superficial reports and assessment procedures had provided the grounds for decisions.

The practical value of this work has been to demonstrate the contribution that a psychoanalytically informed assessment method can make to the sensitivity of decision-making in child care, and thus to the broader therapeutic and consultative roles which child psychotherapists can usefully take. Bradley's work is a model demonstration of how Grounded Theory methods can be used not merely to undertake research analysis on previously completed clinical work, but also as an in vivo component of practical interventions.

I hope that the five examples summarised above have demonstrated the value of grounded theory methods in research in child psychotherapy which advances its knowledge, not merely of the outcomes of treatment, but also of its theories and techniques. One notable feature is that in three out of five of these (those of Anderson, Reid and Bradley) the research was with samples of cases, not on a single case alone, though numbers were unavoidably limited by the fact that these were all single-handed researchers. Where larger numbers of comparable cases can be gathered together (for example as a by-product of a randomised controlled trial outcome study like IMPACT) there is potential for deploying the Grounded Theory method of research with broader comparative aims. 
The broader social science literature on Grounded Theory methods is at present notable for the absence of reference to unconscious mental phenomena. The Handbook of Grounded Theory published by Sage, one of many such texts on research methods, makes scarcely any reference to psychoanalytic ideas. A systematic demonstration of the uses of grounded theory in different contexts of psychoanalytic research - for example in settings of infant observation, young child observation, 'work discussion', clinical work with individuals and groups, and in consultancy to organisations is much needed and long overdue.

\section{References}

Anderson, J. (2003) 'The mythic significance of risk-taking, dangerous, behaviour,' Journal of Child Psychotherapy, 29 (1) 75-91

Anderson, J. (2006) 'Well-suited partners: psychoanalytic research and grounded theory', Journal of Child Psychotherapy, 32, (3,) 329-348

Ball, S.J. (2013)The Education Debate $\left(2^{\text {nd }}\right.$ ed) Bristol: Policy Press.

Black, M. (1962). Models and Metaphors. Ithaca, NY: Cornell University Press.

Boston, M. and Szur, R. (1983) Psychotherapy with Severely Deprived Children.

London: Routledge

Briggs, A. (ed) (2012) Waiting to be Found: Papers on Children in Care. London:

Karnac.

Britton, R. (1998) 'The analyst's intuition: selected fact or overvalued idea?' in Belief and Imagination, London: Routledge. Pp 97-198.

Britton, R. (2015) Between Mind and Brain: Models of the Mind: Models in the Mind. London: Karnac. Pp 45-54

Charmaz, K. (2006) Constructing Grounded Theory. London: Sage.

Clarke, J. and Newman, J. (1997). The Managerial State. London: Sage.

Clarke, J.H., Gewirtz, S, McLaughlin, E. (eds) (2000) New Managerialism, New

Welfare. London: Sage.

Fonagy, P. and Target, M. (2003) Psychoanalytic Theories: Perspectives from

Developmental Psychology. 
Fonagy, P. (200) 'Research in child psychotherapy: Programmes, problems and possibilities', in N. Midgley, J. Anderson, E. Grainger, T. Nesic-Vuckovic, C. Urwin (eds.) Child Psychotherapy and Research. London: Routledge.

Galison, P. \& Stump, D.J. (eds) (1996) The Disunity of Science. Stanford, Ca:

Stanford University Press

Geertz, C. (1973) The Interpretation of Cultures. New York: Basic Books

Glaser, B. and Strauss, A. (1967) The Discovery of Grounded Theory.

Grünbaum, A. (1974) The Foundations of Psychoanalysis: a Philosophical Critique.

Berkeley, Ca: University of California Press.

Heimann, P. (1950) 'On counter-transference,' International Journal of

Psychoanalysis, 31 (1/2) 81-84

Henry, G. (1974). 'Doubly Deprived'. Journal of Child Psychotherapy,3 (4) 29-43.

Hinshelwood, R.D. (2013) Research on the Couch: single case-studies, subjectivity and scientific knowledge. London: Routledge.

Hoggart, R. (1967) The Uses of Literacy. London: Chatto and Windus. Pp 291-317.

Keegan, J. (1976) The Face of Battle: Agincourt, Waterloo and the Somme. London: Jonathan Cape.

Kennedy, E. (2004) Child and Adolescent Psychotherapy: A Systemic Review of Psychoanalytic Approaches. North Central London Strategic Health Authority. Kennedy, E., and Midgley, N. (2007) Process and Outcome Research in Child, Adolescent, and Parent-Infant Psychotherapy: a Thematic Review. North Central London Strategic Health Authority.

Klein, M. (1926) 'The Psychological Principles of Infant Analysis', reprinted in Love Guilt and Reparation and other works 1921-1945. London: Hogarth Press (1975) pp $128-138$.

Kuhn, T.S. (1962) The Structure of Scientific Revolutions. Chicago, III. Chicago University Press.

Kuhn, T.S. (2000)The Road Since Structure: Philosophical Essays 1970-1993.

Chicago: Chicago University Press.

Latour, B. (1983) Science in Action. Cambridge, Ma: Harvard University Press. Layard, R. and Clarke, D. (2015) Thrive: The Power of Psychological Therapy. London: Penguin.

Le Grand, J. (2006) Motivation, Agency, and Public Policy: Of Knights and Knaves, Pawns and Queens. Oxford: Oxford University Press.

Meltzer, D. (1988) 'Aesthetic Conflict and its place in Development', in D. Meltzer and M.Harris Williams, The Apprehension of Beauty. Perthshire: Clunie Press, pp 734. 
Midgley, N. (2004) 'Sailing between Scylla and Charybdis; incorporating qualitative approaches into child psychotherapy research.' Journal of Child Psychotherapy 30 (1) 89-111.

Midgley, N. (2006)'The inseparable bond between cure and research': clinical case study as a method of psychoanalytic inquiry.' Journal of Child Psychotherapy 32 (2) 122-147.

Midgley, N. and Kennedy, E., (2011) 'Psychodynamic psychotherapy for children and adolescents: a critical review of the evidence base.' Journal of Child Psychotherapy 37, (3) 232-260.

Money-Kyrle, R. (1968).'Cognitive Development'. International Journal of Psychoanalysis 49, 691-698.

Money, Kyrle, R. (1971) 'The Aim of Psychoanalysis'. International Journal of Psychoanalysis, 52, 103-106.

Popper, K.R. (1963) Conjectures and Refutations. London: Routledge and Kegan pp 35-65.

Puttnam, H. (1975) 'The Corroboration of Theories', in Mathematics, Matter and Method: Philosophical Papers Vol.1 Cambridge: Cambridge University Press. Pp 250-269.

Reid, M. (2003) 'Clinical Research: the inner world of the mother and her new baby: born in the shadow of death.' Journal of Child Psychotherapy, 29 (2) 207-226 Ricoeur, P. (1970). Freud and Philosophy. New Haven, Conn: Yale University Press. Pp 345-375.

Rosenfeld, H. (1971) 'A clinical approach to the psychoanalytic theory of the life and death instincts: an investigation into the aggressive aspects of narcissism', International Journal of Psychoanalysis, 52: 169-178.

Rustin, M. J. (2014) Review of R.D. Hinshelwood, Research on the Couch (2013) Journal of Child Psychotherapy, Vol 40, (1) 118-126

Shallcross, W. (2012) 'What can be learned from a single case of infant observation. In C. Urwin and J. Sternberg, Infant Observation and Research: Emotional Processes in Everyday Lives. London: Routledge. Pp 69-80.

Spence, D.P. (1994) 'The special nature of psychoanalytic facts.' International Journal of Psychoanalysis. 75, (5/6), 915-927.

Stove, D. (1982) Popper and After: Four Modern Irrationalists. Oxford: Pergamon Press.

Strachey, J. (1934) 'The nature of the therapeutic action of psychoanalysis,' International Journal of Psychoanalysis, 15 pp 127 - 159. 
Kuhn, T.S. (2000) 'The Road Since Structure: Philosophical Essays 1970-1993.

Chicago: Chicago University Press. Pp 90-104.

Tuckett, D. (1994) 'Developing a Grounded Hypothesis to Understand a Clinical

Process: The Role of Conceptualisation in Validation' International Journal of Psycho-Analysis, 1994; v. 75, p1159, 22p

Tuckett, D. (2000) 'Theoretical Pluralism and the Construction of Psychoanalytic Knowledge,' in P. Fonagy, R. Michels, J. Sandler (ed) Changing Ideas In A Changing World: The Revolution in Psychoanalysis. Essays in Honour of Arnold Cooper. London: Karnac.

Tuckett, D. (2005) 'Some Developments in Psychoanalytic Thinking in Great Britain and Continental Europe' in M. Cooper, G. Gabbard, E. Person (eds) American Psychiatric Publishing Textbook of Psychoanalysis. Washington DC and London: American Psychiatric Publishing.

Wakelyn, J. (2011) 'Therapeutic observation of an infant in foster care. 'Journal of Child Psychotherapy 37 (3) 280 - 310).

Wallerstein, R. (2005) 'Will psychoanalytic pluralism be the enduring state of our discipline?' International Journal of Psychoanalysis, 86, (3) 623-6.

Weber, Max. (1978) Economy and Society, Vol 1. Berkeley Ca: University of California Press.

Zizek, S. (1990) 'Eastern European Republics of Gilead.' New Left Review 183.

Oct-Nov., pp 50-62. 